\title{
TWO PROBLEMS ASSOCIATED WITH CONVEX FINITE TYPE DOMAINS
}

\author{
Alexander Iosevich, Eric Sawyer and Andreas Seeger
}

\begin{abstract}
We use scaling properties of convex surfaces of finite line type to derive new estimates for two problems arising in harmonic analysis. For Riesz means associated to such surfaces we obtain sharp $L^{p}$ estimates for $p>4$, generalizing the Carleson-Sjölin theorem. Moreover we obtain estimates for the remainder term in the lattice point problem associated to convex bodies; these estimates are sharp in some instances involving sufficiently flat boundaries.
\end{abstract}

\section{Introduction}

Let $\Omega$ be a convex domain in $\mathbb{R}^{d}$ with smooth boundary. We assume that $\partial \Omega$ is of finite line type, that is, at each point each tangent line has finite order of contact.

We discuss two problems in this paper. Both problems have in common that progress can be made using some approximate scaling properties of $\partial \Omega$. We derive an extension of the Carleson-Sjölin theorem concerning $L^{p}$ convergence results for Riesz means defined by a distance function associated to $\Omega$; we assume that $1 \leq p \leq 4 / 3$. We also give asymptotics for the number of integer lattice points inside large dilates of $\Omega$; the bounds for the error terms are sharp in some cases where there exist points with all lines tangent to the boundary having high order of contact with $\partial \Omega$.

\subsection{Riesz means.}

We assume that the origin belongs to the interior of $\Omega$. Let $\rho: \mathbb{R}^{d} \rightarrow$ $[0, \infty)$ homogeneous of degree 1 be the Minkowski functional associated to $\Omega$; i.e. $\rho$ is homogeneous of degree one, so that $\rho(\xi)=1$ if $\xi \in \partial \Omega$. The

2000 Mathematics Subject Classification. 42B, $11 \mathrm{H}$.

Key words. Lattice points, convex bodies, finite type, maximal Riesz means.

Research supported in part by NSF grants. 
boundary $\Sigma_{\rho}:=\partial \Omega$ is then the unit sphere for the generalized distance function $\rho$. The Bochner-Riesz operator associated to $\rho$ is defined by

$$
\widehat{S_{\lambda, \rho} f}(\xi)=(1-\rho(\xi))_{+}^{\lambda} \widehat{f}(\xi) ;
$$

here our definition of the Fourier transform is $\widehat{f}(\xi)=\int f(y) e^{-\imath\langle y, \xi\rangle} d y$. It is well known that if $1 \leq p<\infty$ the $L^{p}$ boundedness of the Bochner-Riesz operator implies $L^{p}$ convergence of the Riesz means $\mathcal{F}^{-1}\left[(1-\rho / t)_{+}^{\lambda} \widehat{f}\right]$ to the limit $f$ if $f \in L^{p}$ and $t \rightarrow \infty$.

A necessary condition for $L^{p}$ boundedness is

$$
\lambda>\lambda(p)=d|1 / p-1 / 2|-1 / 2 .
$$

Indeed in view of the compact support of the multiplier it is necessary for $L^{p}$ boundedness that the inverse Fourier transform of $(1-\rho)_{+}^{\lambda}$ belongs to $L^{p}$. Using standard asymptotic expansions one can show (working near points on $\Sigma_{\rho}$ where the curvature does not vanish) that (1.2) is necessary for $\mathcal{F}^{-1}\left[(1-\rho)_{+}^{\lambda}\right] \in L^{p}$.

It is known $[\mathbf{9}],[\mathbf{2 9}]$ that the validity of an $L^{2}$ restriction theorem for the Fourier transform implies the $L^{p}$ boundedness of the Bochner-Riesz operator. Since $\Sigma_{\rho}$ is of finite type, say $\leq n$, it follows from [3] that the Fourier transform of $\widehat{d \sigma}(\xi)$ of a smooth density carried by $\Sigma_{\rho}$ is $O\left(|\xi|^{-\mu}\right)$ for some $\mu$ with $\mu \geq(d-1) / n$. Using the appropriate versions of the Stein-Tomas restriction theorem $[\mathbf{1 0}]$ one can show that $L^{p}$ boundedness holds for $1 \leq p \leq 2(\mu+1) /(\mu+2)$ and $\lambda>\lambda(p)(c f .[\mathbf{2 9}])$. Note that $2(\mu+1) /(\mu+2)=(2 n+2 d-2) /(2 n+d-1)$ for the example $x_{d}=\sum_{i=1}^{d-1} x_{i}^{n}$ with even $n$, so that the range obtained in this way is small for large $n$.

Theorem 1.1. Suppose that $d \geq 2,1 \leq p \leq 4 / 3, \lambda>d(1 / p-1 / 2)-1 / 2$, and that $\Sigma_{\rho}$ is of finite line type. Then $S_{\lambda, \rho}$ is bounded on $L^{p}\left(\mathbb{R}^{d}\right)$.

It is conjectured that $L^{p}$ boundedness holds for the same range of exponents as for the sphere. The conjecture for the sphere is that $L^{p}$ boundedness should hold for $\lambda>\lambda(p)$ for $p<2 d /(d+1)$. This is currently known only in two dimensions, see [4]. Sjölin $[\mathbf{2 8}]$ extended this result to arbitrary planar domains with smooth boundary, for some variants concerning convex domains in the plane with nonsmooth boundary, see also the more recent paper by Ziesler and the third author [27]. For partial results in higher dimensions, in the case that the Gauß curvature of $\Sigma_{\rho}$ does not vanish, we refer to Bourgain [1] and for background to [29]. Our proof of Theorem 1.1 uses a variant of Córdoba's geometrical proof $[\mathbf{6}]$ of the Carleson-Sjölin theorem and rescaling. 


\subsection{Multitype and an estimate for the Fourier transform of} surface carried measure.

A precise estimate of the Fourier transforms of surface carried measure is due to Bruna, Nagel, and Wainger [3]. Let $\Sigma=\partial \Omega$ and $H_{P}(\Sigma)$ the affine tangent plane at $P \in \Sigma$, and let

$$
B(P, \delta)=\left\{y \in \Sigma: \operatorname{dist}\left(y, H_{P}(\Sigma)\right)<\delta\right\} .
$$

Then

$$
|\widehat{d \sigma}(\xi)| \leq C\left[\left|B\left(P_{+},|\xi|^{-1}\right)\right|+\left|B\left(P_{-},|\xi|^{-1}\right)\right|\right]
$$

where $P_{ \pm}$are the points on $\Sigma$ for which $\xi$ is a normal vector and $|B|$ denotes the surface measure of $B$. For many problems it is important to know not just the size of the balls but also the distribution function of $x \mapsto|B(x, \delta)|$ and how it relates to the notions of multitype and type. We review the definition of multitype which is implicit in [26], see also [17].

Consider a smooth real valued function $\Phi$ defined in a neighborhood of the origin in a $d$-1-dimensional Euclidean vector space $\mathbb{E}^{d-1}$ so that $\Phi(0)=\nabla \Phi(0)=0$. We say that a vector $v$ in $\mathbb{E}^{d-1}$ has contact of order at least $n+1$ if

$$
\Phi(s v)=O\left(s^{n+1}\right) \quad \text { if } s \rightarrow 0 .
$$

The sets

$$
S^{n}=\left\{v \in \mathbb{E}^{n}: v \text { has contact of order at least } n+1\right\}
$$

are linear subspaces of $\mathbb{E}^{d-1}$ and there are even integers $m_{1}, \ldots, m_{k}$ so that $m_{1}<\cdots<m_{k}, 1 \leq k \leq d-1$, and $m_{0}:=m_{1}-1 \geq 1$ and

$$
0=S^{m_{k}} \subsetneq \cdots \subsetneq S^{m_{0}}:=\mathbb{E}^{d-1}
$$

moreover the sequence is maximal, in the sense that $S^{n}=S^{m_{k}}$ if $m_{k-1}<$ $n \leq m_{k}$. Define

$$
\begin{aligned}
a_{i}=m_{j} \quad \text { if } d-1-\operatorname{dim} S^{m_{j-1}}<i \leq d-1-\operatorname{dim} S^{m_{j}}, \\
j=1, \ldots, k .
\end{aligned}
$$

The $d$-1-tuple $\mathfrak{a}=\left(a_{1}, \ldots, a_{d-1}\right)$ is then called the multitype of $\Phi$ at 0 .

To illustrate the above definitions consider a convex body whose boundary passes through the origin and nearby is given by the equation $x_{d}=\sum_{i=1}^{d-1}\left|x_{i}\right|^{a_{i}}$ where the $a_{i}$ are even integers, with $a_{i} \leq a_{i+1}$, $1 \leq i \leq d-2$. In this case the multitype is $\left(a_{1}, \ldots, a_{d-1}\right)$ and the subspaces $S^{m}$ above are $S^{m}=\operatorname{span}\left(\left\{e_{i}: a_{i}>m\right\}\right)$ (and $S^{m}=\{0\}$ if $\left.m \geq a_{d-1}\right)$. 
We now fix $P \in \Sigma$, choose a unit normal $n_{P}$ and parametrize $\Sigma$ near $P$ as a graph over its tangent plane at $P$. Thus the parametrization is given by

$$
v=\Gamma(v) \mapsto P+v+\Phi(v) n_{P}
$$

for $v \in T_{P} \Sigma$, and $\Phi$ is a convex function vanishing of second order at the origin. We perform the above construction for $\Phi(v)$ defined on $\mathbb{E}^{d-1}=T_{P} \Sigma$ and obtain a flag of subspaces

$$
0=S_{P}^{m_{k}} \subsetneq \cdots \subsetneq S_{P}^{m_{0}}=T_{P} \Sigma .
$$

Let $W_{j}$ be the orthogonal complement of $S_{P}^{m_{j}}$ in $S_{P}^{m_{j-1}}, j=1, \ldots, k$, then

$$
T_{P} \Sigma=W_{1} \oplus \cdots \oplus W_{k} .
$$

We denote by $\Pi_{j}^{P}$ the orthonormal projection on $T_{P} \Sigma$ to $W_{j}$. We also have a similar decomposition and projections $\Pi_{j}^{P}$ to $W_{j}^{*}$ on $T_{P}^{*} \Sigma$, here we let $W_{j}^{*}$ the space of linear functionals on $W_{j}$ extended by 0 on the orthogonal complement of $W_{j}$. We can extend these projections to linear maps on $T_{P}^{*} \mathbb{R}^{d} \simeq\left(\mathbb{R}^{d}\right)^{*}$ by defining $\Pi_{j}^{P} n_{P}=0$.

On $T_{P}^{*} \Sigma$ we define a nonisotropic distance function $\rho_{*}$ by

$$
\rho_{*}(\eta)=\sum_{j=1}^{k}\left|\Pi_{j}^{P} \eta\right|^{\frac{m_{j}}{m_{j}-1}} ;
$$

here $|\cdot|$ denotes the Euclidean distance in $W_{j}$. If $\xi \in T_{P}^{*} \mathbb{R}^{d}$ is taken from a suitable conic neighborhood of $n_{P}$ and $\Pi^{P}$ denotes the projection to $T_{P}^{*} \Sigma$ we define

$$
\Theta_{P}(\xi)=\rho_{*}\left(\frac{\Pi^{P} \xi}{\left\langle\xi, n_{P}\right\rangle}\right) .
$$

Finally we set for $l \leq d-2$

$$
\nu_{l}(P)=\sum_{i=l}^{d-1} a_{i}^{-1}=\sum_{j=1}^{k} \frac{\operatorname{dim} S_{P}^{m_{j-1}}-\operatorname{dim} S_{P}^{m_{j}}}{m_{j}}
$$

and write $\nu(P) \equiv \nu_{1}(P)$. An alternative description of $\nu(P)$ (see [16]) is

$$
\nu(P)=\sup \left\{q: \operatorname{dist}\left(\cdot, H_{P} \Sigma\right) \in L^{q}(\Sigma)\right\} ;
$$

in fact for $q=\nu(P)$ the function $\operatorname{dist}\left(\cdot, H_{P} \Sigma\right)^{-1}$ belongs to the space $L^{q, \infty}(\Sigma)$.

Our result for the Fourier transform of surface carried measure is 
Proposition 1.2. Let $P \in \partial \Omega$. Then there is a neighborhood $U$ of $P$ and a conic neighborhood $V$ of $\left\{ \pm n_{P}\right\}$ in $\mathbb{R}^{d}$ so that for all $\chi \in C_{0}^{\infty}(U)$ and all $\xi \in V$ with $|\xi| \geq 1$ we have

$$
|\widehat{\chi d \sigma}(\xi)| \lesssim\|\chi\|_{C^{N}} \min \left\{|\xi|^{-\nu},|\xi|^{-\frac{1}{2}-\nu_{2}}\left[\Theta_{P}(\xi)\right]^{\nu-\nu_{2}-\frac{1}{2}}\right\}
$$

here $\|\chi\|_{C^{N}}=\max _{\alpha \leq N}\left\|\chi^{(\alpha)}\right\|_{L^{\infty}(U)}$ and $N$ is sufficiently large.

In this statement $N>d+m_{k}$ will suffice. Note the proposition is an improvement over previous results only in the case where all the principal curvatures vanish (and thus $a_{1}>2$ ).

\subsection{A lattice point estimate.}

Let

$$
\mathcal{N}_{\Omega}(t)=\operatorname{card}\left(t \Omega \cap \mathbb{Z}^{d}\right)
$$

It is well known (and elementary) that $\mathcal{N}_{\Omega}(t)$ is asymptotic to $t^{d} \operatorname{vol}(\Omega)$ as $t \rightarrow \infty$ and that the error term

$$
E_{\Omega}(t)=\mathcal{N}(t)-t^{d} \operatorname{vol}(\Omega)
$$

is $O\left(t^{d-1}\right)$. Moreover if $\partial \Omega$ has suitable curvature properties then the error term improves; in particular if the Fourier transform of the surface measure on the boundary satisfies $\widehat{d \sigma}(\xi)=O\left(|\xi|^{-\alpha}\right)$ then the classical method (see e.g. [11], [13, Theorem 7.7.16], and [24]) yields $E_{\Omega}(t)=$ $O\left(t^{d-1-\frac{\alpha}{d-\alpha}}\right)$. This estimate however is not sharp, and several authors beginning with van der Corput have obtained improvements for the case of nonvanishing Gauß curvature; see the monographs by Krätzel [18] and Huxley [14], and in particular the papers by Krätzel and Nowak [20] and recent improvements by W. Müller $[\mathbf{2 2}]$ for results on general convex bodies with nonvanishing curvature in higher dimensions. In $[\mathbf{2 4}, \mathrm{I}]$, [25] Randol obtained better estimates for the case of convex domains in the plane with finite type boundary; these are sharp for $\Omega=\{x$ : $\left.x_{1}^{k}+x_{2}^{k} \leq 1\right\}$ where $k \geq 4$ is even. See also [23] for more refined results. Generalizations to domains of the form $\Omega=\left\{x: x_{1}^{k}+\cdots+x_{d}^{k} \leq 1\right\}$ are in $[24, \mathrm{II}],[\mathbf{1 9}]$.

Here we give a version for general convex bodies with finite type boundary in higher dimensions. Let $\nu(P)=\nu_{1}(P)$ and $\nu_{2}(P)$ as in (1.13) above. 
Theorem 1.3. Let

$$
\nu=\min _{P \in \partial \Omega} \nu(P), \quad \mu=\frac{1}{2}+\min _{P \in \partial \Omega} \nu_{2}(P) .
$$

Then there is a constant $C$ depending on $\Omega$ so that

$$
\left|E_{\Omega}(t)\right| \leq C_{\Omega}\left(1+t^{d-1-\nu}+t^{d-1-\frac{\mu}{d-\mu}}\right) .
$$

Specifically, if $\Gamma$ is the set of all points $P \in \partial \Omega$ at which all principal curvatures vanish then

$$
E_{\Omega}(t)=\sum_{P \in \Gamma} t^{d-1-\nu(P)} G_{P}(t)+O\left(t^{d-1-\frac{\mu}{d-\mu}}\right)
$$

where $G_{P}(t)$ is bounded as $t \rightarrow \infty$. If the normal line determined by $n_{P}$ coincides with $\mathbb{R}_{i}$ for some $i \in\{1, \ldots, d\}$ then $\limsup _{t \rightarrow \infty}\left|G_{P}(t)\right|>0$.

We note that the number $\mu /(d-\mu)$ is greater then $(2 d-1)^{-1}$ since $\mu>1 / 2$. In particular if the Gauß curvature only vanishes at one point at the surface and if $\nu<\mu /(d-\mu)$, then there is $A \in S O(d)$ so that $\lim \sup _{t \rightarrow \infty} t^{\nu-d+1}\left|E_{A \Omega}(t)\right|$ is positive (for other model cases compare [19], [23]). (1.18) over $P \in \Gamma$ is finite since $\Gamma$ is a discrete subset of $\partial \Omega$ (as noted in [16], cf. the proof of Lemma 2.2 below). We remark that it is well known that for almost all rotations $A \in S O(d)$ the error terms $E_{A \Omega}(t)$ improve, see [5], [31], [32], [23], [15], and [2].

We shall derive the estimate for the Fourier transform in Proposition 1.2 in the next section. Section 3 contains the application to the lattice point problem. In Section 4 and Section 5 we prove results on Bochner-Riesz multipliers; here we first consider the case of one nonvanishing principal curvature and then in Section 5 the case of convex domains.

Notation. Given two quantities $A, B$ we write $A \lesssim B$ if there is an absolute positive constant $C$ so that $A \leq C B$. We write $A \approx B$ if $A \lesssim B$ and $B \lesssim A$.

Acknowledgement. We thank the referee for pointing out some misprints and for making a suggestion concerning the exposition.

\section{An estimate for Fourier transforms of surface carried measures}

We begin by reviewing some facts about classes of convex functions in $[\mathbf{3}],[\mathbf{2 6}],[\mathbf{1 6}],[\mathbf{1 7}]$.

Let $B_{T} \subset \mathbb{R}^{n}$ denote the open ball of radius $T$ centered at 0 ; it is always assumed that $T \leq 1$. 
Fix a flag $\mathfrak{V}$ of subspaces $0=\mathbb{V}_{k} \subsetneq \cdots \subsetneq \mathbb{V}_{0}$ of $\mathbb{E}^{d-1}$, with $\mathbb{V}_{0}=$ $\mathbb{E}^{d-1}$, and let $m=\left(m_{1}, \ldots, m_{k}\right)$ be a $k$-tuple of even positive integers with $m_{1}<\cdots<m_{k}$. For $0<b \leq M, N \in \mathbb{Z}^{+}, N>m_{k}$, let $\mathfrak{S}_{T}^{d-1}(b, M, \mathfrak{V}, m, N)$ be the class of all $C_{N}\left(\overline{B_{T}}\right)$ functions $g$ with the property that

$$
\begin{array}{ll}
g(0)=\nabla g(0)=0 & \\
\left.\frac{d^{2}}{(d t)^{2}} g(x+t \theta)\right|_{t=0} \geq 0 & \text { for all } \theta \in S^{d-2}, x \in B_{T} \\
\max _{2 \leq j \leq m_{l}}\left|\left(\frac{d}{d t}\right)^{j} g(x+t \theta)\right|_{t=0} \mid \geq b & \text { for all } \theta \in S^{d-2} \cap \mathbb{V}_{l-1}, x \in B_{T} \\
\max _{|\alpha| \leq N}\left|\left(\frac{\partial}{\partial x}\right)^{\alpha} g(x)\right| \leq M & \text { for all } x \in B_{T} .
\end{array}
$$

Here $S^{d-2}$ denotes the unit sphere in $\mathbb{E}^{d}$. We also define $\mathfrak{a}(\mathfrak{V}, m)=$ $\left(a_{1}(\mathfrak{V}, m), \ldots, a_{l}(\mathfrak{V}, m)\right)$ by

$(2.2) \quad a_{i}(\mathfrak{V}, m)=m_{j}(\mathfrak{V}, m) \quad$ if $d-1-\operatorname{dim} \mathbb{V}_{j-1}<i \leq d-1-\operatorname{dim} \mathbb{V}_{j}$,

in analogy to (1.7).

Now if $P \in \Sigma$ (with $\Sigma=\partial \Omega$ as in the introduction) and $\mathbb{E}^{d-1}=T_{P} \Sigma$ then let $\mathbb{V}_{j}=S_{P}^{m_{j}} \subset T_{P} \Sigma$ as in (1.5). Let $\Phi$ be as in (1.8). Then there is $T>0$ and a neigborhood $\mathcal{U}$ of 0 so that for all $w \in \mathcal{U}$ the functions $y \mapsto$ $\Phi(w+y)-\Psi(w)-\left\langle y, \nabla_{w} \Phi(w)\right\rangle$ belong to $\mathfrak{S}_{T}^{n}(b, M, \mathfrak{V}, m, N)$; moreover there are positive constants $c_{0}, C_{0}, C_{1}$ so that

$$
\mathcal{B}(w, \delta)=\left\{y:\left|\Phi(y)-\Phi(w)-\left\langle\nabla_{w} \Phi(w), y-w\right\rangle\right| \leq \delta\right\}
$$

belongs to $B_{T}$ if $\delta \leq c_{0} T^{m_{k}}$ and satisfies

$$
\operatorname{meas}(\mathcal{B}(w, \delta)) \leq C \delta^{\nu} ;
$$

see Proposition 2.1 in $[\mathbf{1 7}]$.

Lemma 2.1. Suppose that $\Phi \in \mathfrak{S}_{T}^{d-1}(b, M, \mathfrak{V}, m, N)$ and suppose that $\mathfrak{a}=\left(a_{1}, \ldots, a_{d-1}\right)$ is the multitype at the origin. Let $\Psi^{w}(y)=\Phi(y)-$ $\Phi(w)-\left\langle\nabla_{w} \Phi(w), y-w\right\rangle$ and let $\mathfrak{a}(w)=\left(a_{1}(w), \ldots, a_{d-1}(w)\right)$ be the multitype of $\Psi^{w}$ at the origin. Then there is a neighborhood $\mathcal{U}$ of the origin so that $a_{i}(w) \leq a_{i}$ for $i=1, \ldots, d-1$ and all $w \in \mathcal{U}$. 
Proof: Let $S^{m_{i}}$ be as in (1.5) and let $\ell>\operatorname{dim} S^{m_{i}}$. Recall that $S^{n}=$ $S^{m_{j-1}}$ for $m_{j}<n \leq m_{j-1}$. Using continuity and compactness arguments together with the definition of the spaces $S^{m_{i}}$ we see that there is a neighborhood $\mathcal{U} \subset \widetilde{\mathcal{U}}$ of the origin so that for every $w \in \widetilde{\mathcal{U}}$, every $y \in \mathcal{U}$ and every $\ell$-tuple of orthonormal vectors $\left\{u_{1}, \ldots, u_{\ell}\right\}$

$$
\sum_{i=1}^{\ell} \sum_{s \leq m_{j}}\left|\left(\left\langle u_{i}, \nabla_{y}\right\rangle\right)^{s} \Psi^{w}(y)\right| \geq b_{0}>0 .
$$

The result of the lemma follows quickly from the definition of the multitype.

We now let $\Sigma$ denote the graph of $\Phi$. On $T_{0} \Sigma=\mathbb{R}^{d-1}$ we define a nonisotropic distance function $\rho$ by

$$
\rho(y)=\sum_{j=1}^{k}\left|\Pi_{j} y\right|^{m_{j}} ;
$$

note that that the unit ball for $\rho^{*}$ in (1.11) is the polar set for the unit ball for $\rho$.

The following lemma gives an improvement of estimates in $[\mathbf{1 6}]$ and [17]. A rescaling argument is used as in those papers; the present improvement is obtained using a more careful argument for the rescaled pieces.

Lemma 2.2. Let $\Phi$ be a convex smooth function defined in a neighborhood of the origin in $\mathbb{R}^{d-1}$, so that $\Phi(0)=\nabla \Phi(0)=0$. Let $\mathfrak{V}$ be the flag of subspaces $\left\{S^{m_{j}}\right\}$ defined as in (1.5). Let $\mathfrak{a}$ be the multitype of $\Phi$ near $0, \mathcal{B}(w, \delta)$ as in (2.3) and $\rho$ as in (2.6). Let $\nu=\sum_{i=1}^{d-1} a_{i}^{-1}$, $\nu_{2}=\sum_{i=2}^{d-1} a_{i}^{-1}$.

Then there is a neighborhood $\mathcal{U}$ of the origin and $\delta_{0}>0$ so that for all $0<\delta \leq \delta_{0}$ and all $w \in \mathcal{U}$

$$
\operatorname{meas}(\mathcal{B}(w, \delta)) \leq C \delta^{\alpha}[\rho(w)]^{\nu-\alpha}, \quad \nu \leq \alpha \leq \frac{1}{2}+\nu_{2} .
$$

Proof: We may assume that $a_{1}>2$ since otherwise the theorem follows already from the estimate (2.4). Let $\left\{u_{1}, \ldots, u_{d-1}\right\}$ an orthonormal basis of $\mathbb{R}^{d-1}$ so that

$$
S^{m_{j}}=\operatorname{span}\left\{u_{i}, d-1-\operatorname{dim} S^{m_{j}}<i \leq d-1\right\}
$$

for $j=0, \ldots, k-1$. By performing a rotation we may assume that the $u_{i}$ are the standard coordinate vectors. 
Define dilations $A_{t}$ by

$$
A_{t} x=\left(t^{\frac{1}{a_{1}}} x, \ldots, t^{\frac{1}{a_{d-1}}} x\right) .
$$

According to $[\mathbf{2 6}],[\mathbf{1 6}]$ we may split

$$
\Phi(x)=Q(x)+R(x)
$$

where $Q$ is a convex polynomial satisfying

$$
Q\left(A_{t} x\right)=t Q(x)
$$

and

$$
0<|Q(x)| \leq C_{1}|x||\nabla Q(x)| \leq C_{2}|x|^{2} \sum_{i, j}\left|\frac{\partial^{2} Q}{\partial x_{i} \partial x_{j}}(x)\right|,
$$

and the remainder term $R$ satisfies

$$
\left|s^{-1} \frac{\partial^{|\alpha|}}{\partial x^{\alpha}}\left(R\left(A_{s} x\right)\right)\right| \lesssim s^{1 / m}
$$

for $|x| \leq T$ and all multiindices $\alpha=\left(\alpha_{1}, \ldots, \alpha_{d-1}\right)$ with $|\alpha| \leq N$. Since $Q$ is positive away from the origin and homogeneous with respect to dilations $\left(A_{t}\right)$ we have that

$$
Q(y) \approx \rho(y)
$$

where $\rho$ is as in $(2.6)$; in fact $\rho(y) \approx \sum_{i=1}^{d-1}\left|\left\langle y, u_{i}\right\rangle\right|^{a_{i}}$.

Set $\Phi_{\ell}(y)=2^{\ell} \Phi\left(A_{2^{-\ell}} y\right)$ and note that $\Phi_{\ell}(y)=Q(y)+R_{\ell}(y)$, where $R_{\ell}$ and its derivatives tend to zero uniformly on compact sets, as $\ell \rightarrow \infty$.

Denote by $\mathfrak{a}(w)=\left(a_{1}(w), \ldots, a_{d-1}(w)\right)$ the multitype of $Q$ at $w$. Then $\mathfrak{a}(0)=\mathfrak{a}$ and by Lemma 2.1 there is $M>0$ so that $a_{i}(w) \leq a_{i}$ for $0 \leq \rho(w) \leq 2^{-M+2}$ and, by $(2.10 / 11), a_{1}(w)=2$ for $0<\rho(w) \leq 2^{-M+2}$; note that nothing is said about the position of the spaces $S^{m}(w)$. Now for any point $w$ there is an open ball $U(w)$ of radius $T(w) / 4$ and a flag $\mathfrak{V}(w)$ consisting of $l(w)$ nested subspaces and an $l(w)$-tuple $m(w)$ so that for $x \in U(w)$ the functions

$$
h \mapsto Q^{x}(h)=Q(x+h)-Q(x)-\langle\nabla Q(x), h\rangle
$$

belong to a class $\mathfrak{S}_{T(w)}^{d-1}(b(w), M(w), \mathfrak{V}(w), m(w), N)$ so that $a_{i}(\mathfrak{V}(w)$, $m(w)) \geq a_{i}$ and $a_{1}(\mathfrak{V}(w), m(w))=2$.

By the metric property of the nonisotropic balls $\mathcal{B}(w, \delta)$ there are constants $C_{2} \gg C_{1} \gg 1$ and $\delta_{1} \ll 1$ so that

$$
\mathcal{B}(y, \delta) \subset\left\{x: C_{1}^{-1} \rho(y) \leq \rho(x) \leq C_{1} \rho(y)\right\} \quad \text { if } \rho(y) \geq C_{2} \delta ;
$$

we may assume that $C_{1} \geq 2^{2 M+4}$. 
We shall now show that there are constants $c_{0}>0, C_{0}>1$ so that for $2^{-\ell} \leq c_{0}$

$$
\begin{aligned}
|\mathcal{B}(y, \delta)| \leq \delta^{\alpha} 2^{\ell(\alpha-\nu)} \quad \text { if } 2^{-l-M} & \leq \rho(y) \leq 2^{-l-M+1} \\
\delta & \leq C_{0}^{-1} 2^{-M-\ell}, 0 \leq \alpha \leq \nu_{2}+\frac{1}{2}
\end{aligned}
$$

Let

$$
W=\left\{y: C_{1}^{-2} 2^{-M-2} \leq \rho(y) \leq C_{1}^{-1} 2^{-M+2}\right\}
$$

which because of $C_{1} \geq 2^{2 M+4}$ is contained in the open ball of radius $2^{-M}$ centered at the origin. We may cover the compact annulus $W$ by finitely many open balls $U_{i}$ with center $w_{i} \in W$ and radius $T\left(w_{i}\right) / 4$ so that $Q^{x} \in \mathfrak{S}_{T\left(w_{i}\right)}^{d-1}\left(b\left(w_{i}\right), M\left(w_{i}\right), \mathfrak{V}\left(w_{i}\right), m\left(w_{i}\right), N\right)$ provided that $\left|x-w_{i}\right| \leq$ $T\left(w_{i}\right) / 2$.

Since $\Phi_{\ell}$ converges to $Q$ in the $C^{N}$-topology uniformly on compact sets, there is a positive constant $c_{0}$ so that for $2^{-\ell} \leq c_{0}$ the functions

$$
h \mapsto \Phi_{\ell}(x+h)-\Phi_{\ell}(x)-\left\langle\nabla \Phi_{\ell}(x), h\right\rangle
$$

belong to $\mathfrak{S}_{T\left(w_{i}\right)}^{d-1}\left(\frac{b\left(w_{i}\right)}{2}, 2 M\left(w_{i}\right), \mathfrak{V}\left(w_{i}\right), m\left(w_{i}\right), N\right)$ if $\left|x-w_{i}\right| \leq T\left(w_{i}\right) / 2$. By the finite type property there is a $\delta_{0}>0$ so that for $\gamma \leq \delta_{0}$ and $x \in U_{i}$ the caps

$$
W_{\ell}(x, \gamma) \subset\left\{z:\left|\Phi_{\ell}(z)-\Phi_{\ell}(x)-\left\langle\nabla \Phi_{\ell}(x), z-x\right\rangle\right| \leq \gamma\right\}
$$

are contained in the double of $U_{i}$; moreover we have

$$
\left|W_{\ell}(x, \gamma)\right| \leq C \gamma^{\frac{1}{2}+\nu_{2}}, \quad \gamma \leq \delta_{0},
$$

by the analogue of (2.4) with exponent $1 / 2+\nu_{2}$; here $C$ is independent of $\ell$.

Now in order to show that (2.13) holds we assume that $C_{1}^{-1} 2^{-l-M} \leq$ $\rho(y) \leq C_{1}^{-1} 2^{-l-M+1}$ and observe that the image of $\mathcal{B}(y, \delta)$ under the linear transformation $A_{2^{\ell}}$ is $W_{\ell}\left(A_{2^{\ell}} y, 2^{\ell} \delta\right)$ which is contained in $W$, in fact in a $U_{i}$ if $2^{\ell} \delta \leq \delta_{0}$. Since $\operatorname{det} A_{2^{\ell}}=2^{\ell \nu}$ we have $|\mathcal{B}(y, \delta)| \lesssim$ $2^{-\ell \nu}\left|W_{\ell}\left(A_{2^{\ell}} y, 2^{\ell} \delta\right)\right|$ and (2.13) follows.

Finally if $\delta \leq C_{0}^{-1} 2^{-M-\ell}$ we use $|\mathcal{B}(y, \delta)|=O\left(\delta^{\nu}\right)$ instead and observe that in this range $\delta^{\alpha} 2^{\ell(\alpha-\nu)} \lesssim \delta^{\nu}$, provided that $\alpha \geq \nu$. This together with (2.13) proves the asserted statement. 
Lemma 2.3. Let $\Phi, \mathfrak{V}, \mathfrak{a}, \mathcal{U}$ be as in Lemma 2.2, $N>d+a_{d-1}$. For $\xi \in \mathbb{R}^{d}$ define

$$
F(\xi)=\int \chi(y) e^{-\imath\left(\left\langle\xi^{\prime}, y\right\rangle+\xi_{d} \Phi(y)\right)} d y .
$$

There is a neighborhood $\widetilde{\mathcal{U}} \subset \mathcal{U}$ of the origin and a conic neighborhood $\mathcal{V}$ of $e_{d}$ so that for $\xi \in \mathcal{V}$

$$
|F(\xi)| \leq C\|\chi\|_{C^{N}}|\xi|^{-\alpha}\left(\sum_{j}\left[\frac{\Pi_{j} \xi}{\left|\xi_{d}\right|}\right]^{\frac{m_{j}}{m_{j}-1}}\right)^{\nu-\alpha} \quad, \quad \nu \leq \alpha \leq \frac{1}{2}+\nu_{2} .
$$

Proof: We may assume that (2.7) holds and that the $u_{i}$ 's form the standard basis in $\mathbb{R}^{d-1}$. Observe that then

$$
\sum_{j}\left|\Pi_{j} \eta\right|^{\frac{m_{j}}{m_{j}-1}} \approx \sum_{i=1}^{d-1}\left|\xi_{i}\right|^{a_{i}^{\prime}}
$$

with $a_{i}^{\prime}=a_{i} /\left(a_{i}-1\right)$.

Assume that $s / 2 \leq \rho(x) \leq 2 s$ and $s$ is small. Then $\left|A_{1 / s} x\right| \approx 1$ and $\left|Q_{x_{i}}\left(A_{1 / s} x\right)\right| \leq C$. But $Q_{x_{i}}\left(A_{1 / s} x\right)=s^{-1+1 / a_{i}} Q_{x_{i}}(x)$ so that $\left|Q_{x_{i}}(x)\right| \lesssim$ $s^{1-\frac{1}{a_{i}}}$. Similarly by $(2.11)$ the remainder term $R_{x_{i}}$ satisfies the same estimate so that

$$
\left|\Phi_{x_{i}}(x)\right| \lesssim\left(\sum_{k=1}^{d-1}\left|x_{k}\right|^{a_{k}}\right)^{1-\frac{1}{a_{i}}}
$$

for small $x$ and therefore

$$
\sum_{i=1}^{d-1}\left|\Phi_{x_{i}}(x)\right|^{a_{i}^{\prime}} \lesssim \sum_{k=1}^{d-1}\left|x_{k}\right|^{a_{k}}
$$

Now let $x(\xi)$ be the unique point at which $\xi$ is normal to the graph of $\Phi$. By the Bruna-Nagel-Wainger estimate for the Fourier transform (1.4) and Lemma 2.2 we have that

$$
|F(\xi)| \lesssim|\xi|^{-\alpha} \rho(x(\xi))^{\nu-\alpha}
$$

and since $x(\xi)$ is determined by $\xi_{i} / \xi_{d}= \pm \Phi_{x_{i}}(x(\xi))$ for $i=1, \ldots, d-1$, the estimate (2.17) follows.

\section{Lattice point estimates}

In this section we prove Theorem 1.3. We use a variant of the classical proof (see [24] for the two-dimensional case). Choose $\zeta \in C_{0}^{\infty}\left(\mathbb{R}^{d}\right)$ so that $\zeta$ is nonnegative, $\zeta(x)=0$ if $|x| \geq 1$, and $\int \zeta(x) d x=1$. Define 
$\zeta_{\varepsilon}(x)=\varepsilon^{-d} \zeta\left(\varepsilon^{-1} x\right)$. We work with the $\varepsilon$-regularization $\chi_{\Omega} * \zeta_{\varepsilon}$ of the characteristic function of $\Omega$ and define

$$
\mathcal{N}_{\varepsilon}(t)=\sum_{k \in \mathbb{Z}^{d}} \chi_{t \Omega} * \zeta_{\varepsilon t}(k) .
$$

By the Poisson summation formula

$$
\begin{aligned}
\mathcal{N}_{\varepsilon}(t) & =\sum_{k \in \mathbb{Z}^{d}} t^{d} \widehat{\chi_{\Omega}}(2 \pi t k) \widehat{\zeta}(2 \pi \varepsilon t k) \\
& =t^{d} \operatorname{vol}(\Omega)+\mathcal{R}_{\varepsilon}(t)
\end{aligned}
$$

where

$$
\mathcal{R}_{\varepsilon}(t)=\sum_{k \neq 0} t^{d} \widehat{\chi_{\Omega}}(2 \pi t k) \widehat{\zeta}(2 \pi \varepsilon t k) .
$$

By the divergence theorem

$$
\widehat{\chi_{\Omega}}(\xi)=\int_{\Omega} e^{-\imath\langle x, \xi\rangle} d x=\imath \sum_{i=1}^{d} \frac{\xi_{i}}{|\xi|^{2}} F_{i}(\xi)
$$

where

$$
F_{i}(\xi)=\int_{\Sigma} n_{i}(y) e^{-\imath\langle y, \xi\rangle} d \sigma(y)
$$

and $n_{i}$ denotes the $i^{\text {th }}$ component of the outer normal vector $n_{P}$.

Let $\Gamma$ be the set of points $P \in \Sigma$ at which all principal curvatures vanish. As noticed in [16] it follows from $(2.10 / 11)$ that the set $\Gamma$ is discrete, thus finite by compactness. For every $P \in \Gamma$ we choose a narrow conic symmetric neighborhood $V_{P}$ of the normals $\left\{ \pm n_{P}\right\}$, a small neighborhood $U_{P}$ of $P$ in $\Sigma$, and a $C_{0}^{\infty}$ function $\chi_{P}$ whose restriction to $\Sigma$ vanishes off $\mathcal{U}$ and so that $\chi_{P}$ equals one in a neighborhood of $P$. We may arrange these neighborhoods so that the sets $\bar{V}_{P} \cap\{\xi:|\xi| \geq 1\}$, $P \in \Gamma$ are pairwise disjoint, and that the normals to all points in a neighborhood of $\bar{U}_{P}$ are contained in $V_{P}$ (thus the $\bar{U}_{P}$ 's are disjoint too).

Define

$$
F_{i, P}(\xi)=\int_{\Sigma} \chi_{P}(y) n_{i}(y) e^{-\imath\langle y, \xi\rangle} d \sigma(y) .
$$

If the cones $V_{P}$ are chosen sufficiently narrow, we have

$$
F_{i, P}(\xi) \lesssim \begin{cases}\min \left\{|\xi|^{-\nu(P)}, \xi^{-\left(\frac{1}{2}+\nu_{2}(P)\right)}\right\} \Theta_{P}\left(\frac{\Pi^{P} \xi}{\left\langle n_{P}, \xi\right\rangle}\right) & \text { if } \xi \in V_{P} \\ C_{N}|\xi|^{-N} & \text { if } \xi \notin V_{P}\end{cases}
$$


The estimate for $\xi \in V_{P}$ follows from Proposition 1.2, and the estimate for $\xi \notin V_{P}$ follows by a simple integration by parts; namely if $t \mapsto \gamma(t)$ parametrizes $\Sigma$ near $P$ then $\left|\left\langle\gamma^{\prime}(t), \xi\right\rangle\right| \approx|\xi|$ for $\gamma(t) \in U_{P}$ and $\xi \notin V_{P}$.

Moreover by the Bruna-Nagel-Wainger estimate we have

$$
\left|F_{i}(\xi)-\sum_{P \in \Gamma} F_{i, P}(\xi)\right| \lesssim|\xi|^{-\mu}, \quad \mu=\frac{1}{2}+\inf _{P \in \Sigma} \nu_{2}(P) ;
$$

here we used the definition of $\Gamma$ and the fact that $\chi_{P}$ equals one near $P$.

We now estimate the remainder term $R_{\varepsilon}(t)$ where $\varepsilon \ll 1 / t$ will be suitably chosen. Let dist $_{\infty}$ denote the distance taken with respect to the $\ell^{\infty}$ metric in $\mathbb{R}^{d}$, or $\mathbb{Z}^{d}$. For $P \in \Gamma$ let

$$
\begin{aligned}
\mathcal{A}_{P} & =\left\{k \in V_{P} \cap \mathbb{Z}^{d}: k \neq 0, \operatorname{dist}_{\infty}\left(k, \mathbb{R} n_{P}\right) \leq 3 / 4\right\} \\
\mathcal{B}_{P} & =\left\{k \in V_{P} \cap \mathbb{Z}^{d}: k \neq 0, \operatorname{dist}_{\infty}\left(k, \mathbb{R} n_{P}\right)>3 / 4\right\} \\
\mathcal{C} & =\left\{k \in \mathbb{Z}^{d}: k \neq 0, k \notin \cup_{P \in \Gamma} V_{P}\right\} .
\end{aligned}
$$

Let

$$
\begin{aligned}
\mathfrak{A}_{P}^{i}(t) & =\sum_{k \in \mathcal{A}_{P}} t^{d} \widehat{\zeta}(2 \pi \varepsilon t k) \frac{2 \pi k_{i}}{|2 \pi k|^{2}} F_{i, P}(2 \pi t k) \\
\mathfrak{B}_{P}^{i}(t) & =\sum_{k \in \mathcal{B}_{P}} t^{d} \widehat{\zeta}(2 \pi \varepsilon t k) \frac{2 \pi k_{i}}{|2 \pi k|^{2}} F_{i, P}(2 \pi t k) \\
\mathfrak{C}_{P}^{i}(t) & =\sum_{k \in \mathcal{C}} t^{d} \widehat{\zeta}(2 \pi \varepsilon t k) \frac{2 \pi k_{i}}{|2 \pi k|^{2}} F_{i, P}(2 \pi t k) \\
\mathfrak{D}^{i}(t) & =\sum_{k \neq 0} t^{d} \widehat{\zeta}(2 \pi \varepsilon t k) \frac{2 \pi k_{i}}{|2 \pi k|^{2}}\left(F_{i}(2 \pi t k)-\sum_{P \in \Gamma} F_{i, P}(2 \pi t k)\right)
\end{aligned}
$$

then

$$
\mathcal{R}_{\varepsilon}(t)=\sum_{i=1}^{d}\left(\mathfrak{D}^{i}(t)+\sum_{P \in \Gamma}\left(\mathfrak{A}_{P}^{i}(t)+\mathfrak{B}_{P}^{i}(t)+\mathfrak{C}_{P}^{i}(t)\right)\right) .
$$

When evaluating $\mathfrak{A}_{P}^{i}$ we essentially sum over integers in a tubular neighborhood of a line and by the estimate (2.4) we certainly get

$$
\left|\mathfrak{A}_{P}^{i}(t)\right| \lesssim \sum_{k \in \mathcal{A}_{P}} t^{d}|t k|^{-1-\nu} \lesssim t^{d-1-\nu}
$$


Next for the estimation of $\mathfrak{D}_{P}^{i}$ we use the rapid decay estimate in (3.4) to obtain

$$
\left|\mathfrak{D}_{P}^{i}(t)\right| \lesssim \sum_{k \neq 0} t^{d}|t k|^{-N} \lesssim t^{d-N}
$$

and for $\mathfrak{C}_{P}^{i}$ we use (3.5) which yields

$$
\left|\mathfrak{C}_{P}^{i}(t)\right| \lesssim C_{N} \sum_{k \neq 0} t^{d}(1+|\varepsilon t k|)^{-N}(1+|t k|)^{-\mu-1} \lesssim \varepsilon^{\mu+1-d} .
$$

Finally

$$
\left|\mathfrak{B}_{P}^{i}(t)\right| \lesssim \sum_{\substack{k \neq 0 \\ k \in V_{P}}} t^{d}|t k|^{-\frac{3}{2}-\nu_{2}(P)} \Theta_{P}\left(\frac{\Pi^{P} k}{\left\langle k, n_{P}\right\rangle}\right)(1+|\varepsilon t k|)^{-N}
$$

and we claim that for $\lambda \geq 1$

$$
\begin{aligned}
\sum_{\substack{|k| \approx \lambda \\
k \in V_{P}}} t^{d}|t k|^{-\frac{3}{2}-\nu_{2}(P)} \Theta_{P}\left(\frac{\Pi^{P} k}{\left\langle k, n_{P}\right\rangle}\right) & (1+|\varepsilon t k|)^{-N} \\
& \lesssim \lambda^{d-\frac{3}{2}-\nu_{2}(P)} \min \left\{1,(\lambda \varepsilon t)^{-N}\right\}
\end{aligned}
$$

which implies

$$
\left|\mathfrak{B}_{P}^{i}(t)\right| \lesssim \varepsilon^{\frac{3}{2}+\nu_{2}(P)-d} \lesssim \varepsilon^{-(d-1-\mu)} .
$$

We verify (3.10). Let $\mathfrak{a}=\mathfrak{a}(P)$ be the multitype at $P$. In view of $\operatorname{dist}\left(k, \mathbb{R} n_{P}\right) \geq 3 / 4$ it is straightforward to check that

$$
\Theta_{P}\left(\frac{\Pi^{P} k}{\left\langle k, n_{P}\right\rangle}\right) \approx \Theta_{P}\left(\frac{\Pi^{P} \xi}{\left\langle\xi, n_{P}\right\rangle}\right) \quad \text { if }|\xi-k|_{\infty} \leq 1 / 2, k \in \mathcal{B}_{P} .
$$

Thus we may replace the sum in (3.10) by an integral. After performing a suitable rotation in this integral we have to show that

$$
\begin{array}{r}
\int_{\left|\xi_{d}\right| \approx \lambda} \int_{\left|\xi^{\prime}\right| \leq \lambda}|\xi|^{-3 / 2-\nu_{2}(P)}\left(\sum_{i=1}^{d-1} \frac{\left|\xi_{i}\right|^{a_{i}^{\prime}}}{|\xi|^{a_{i}^{\prime}}}\right)^{\nu-\nu_{2}(P)-\frac{1}{2}} d \xi^{\prime} d \xi_{d} \\
\lesssim \lambda^{d-\frac{3}{2}-\nu_{2}(P)} .
\end{array}
$$

Now $\left(\sum_{i=1}^{d-1}\left(\left|\xi_{i}\right| /|\xi|\right)^{a_{i}^{\prime}}\right)^{\nu-\nu_{2}-\frac{1}{2}} \lesssim\left(\left|\xi_{1}\right| /|\lambda|\right)^{a_{1}^{\prime}\left(1 / a_{1}-1 / 2\right)}$ with $a_{1}^{\prime}\left(1 / a_{1}-\right.$ $1 / 2)>-1$, and therefore the integral in (3.12) is bounded by

$$
\lambda^{d-1-3 / 2-\nu_{2}(P)} \int_{\left|\xi_{1}\right| \leq \lambda}\left(\left|\xi_{1}\right| /|\lambda|\right)^{a_{1}^{\prime}\left(1 / a_{1}-1 / 2\right)} d \xi_{1} \lesssim \lambda^{d-3 / 2-\nu_{2}(P)} .
$$

This shows (3.10). 
To finish the proof we note that

$$
N_{\varepsilon}(t(1-C \varepsilon)) \leq \mathcal{N}_{\Omega}(t) \leq N_{\varepsilon}(t(1+C \varepsilon))
$$

where $C$ is a constant depending only on the geometry of $\Omega$. Thus, by taking into account the leading term in (3.1) we see that

$$
E_{\Omega}(t) \lesssim\left(t^{d-1-\nu}+t^{d} \varepsilon+\varepsilon^{-(d-1-\mu)}\right)
$$

and the desired estimate follows if we choose $\varepsilon=t^{-d /(d-\mu)}$. This completes the proof of (1.17).

Lower bounds: To show (1.18) we work with our choice $\varepsilon=\varepsilon(t)=$ $t^{-d /(d-\mu)}$. For (1.18) we simply set

$$
G_{P}(t)=\sum_{i=1}^{d} t^{\nu(P)-d-1} \mathfrak{A}_{P}^{i}(t)
$$

which we already showed to be bounded above. However we have to verify the claim that $\lim \sup _{t \in \infty}\left|G_{P}(t)\right|>0$ in the case where $n_{P}= \pm e_{i}$.

We now assume that $n_{P}=e_{i}$ (the case $n_{P}=-e_{i}$ is handled in the same way). Then define

$$
\begin{aligned}
& G_{P}(t) \\
= & t^{\nu(P)+1-d} \sum_{\kappa \in \mathbb{Z} \backslash\{0\}}(2 \pi)^{-1} t^{d} \widehat{\zeta}\left(2 \pi t^{-\mu /(d-\mu)} \kappa e_{i}\right) \operatorname{sign}(\kappa)|\kappa|^{-1} F_{i, P}\left(2 \pi t \kappa e_{i}\right) .
\end{aligned}
$$

We split this sum into parts $G_{P}(t)=I(t)+I I(t)$ where

$$
\begin{aligned}
& I_{P}(t) \\
& =(2 \pi)^{-1} t^{\nu(P)+1} \sum_{\kappa \in \mathbb{Z} \backslash\{0\}} \operatorname{sign}(\kappa)|\kappa|^{-1} F_{i, P}\left(2 \pi t \kappa e_{i}\right) \\
& I I_{P}(t) \\
& =(2 \pi)^{-1} t^{\nu(P)+1} \sum_{\kappa \in \mathbb{Z} \backslash\{0\}}\left(1-\widehat{\zeta}\left(2 \pi t^{-\mu /(d-\mu)} \kappa e_{i}\right)\right) \operatorname{sign}(\kappa)|\kappa|^{-1} F_{i, P}\left(2 \pi t \kappa e_{i}\right) .
\end{aligned}
$$

For the estimation of $I I$ we note that $\left|\left(1-\widehat{\zeta}\left(2 \pi t^{-\mu /(d-\mu)} \kappa e_{i}\right)\right)\right| \lesssim$ $\min \left\{1, t^{-\mu /(d-\mu) \kappa}\right\}$ with and since $F_{i, P}\left(2 \pi t \kappa e_{i}\right)=O\left((t \kappa)^{-\nu}\right)$ we get the estimate

$$
|I I(t)| \lesssim t^{-\frac{\mu}{d-\mu}}
$$

To examine $I(t)$ we parametrize by our assumption on $n_{P}=e_{i}$.

$$
F_{i, P}\left(2 \pi t \kappa e_{i}\right)=e^{-\imath \kappa\left\langle P, e_{i}\right\rangle} \int_{y^{\prime} \in \mathbb{R}^{d-1}} \chi_{0}\left(y^{\prime}\right)\left(1+\left|\nabla \Phi\left(y^{\prime}\right)\right|^{2}\right)^{1 / 2} e^{\imath \kappa \Phi\left(y^{\prime}\right)} d y^{\prime}
$$


where $\Phi \equiv \Phi^{P}$ is convex, vanishes of second order at the origin of $\mathbb{R}^{d-1}$ and has multitype $\mathfrak{a}(P)$ there; $\chi_{0}$ is smooth, compactly supported and equal to one in a neighborhood of the origin. By the convexity $\left\langle P, n_{P}\right\rangle=$ $\left\langle P, e_{i}\right\rangle \neq 0$. To examine the integral we may use an asymptotic expansion derived in $[\mathbf{2 6}]$ (stated there for $\kappa \rightarrow \infty$, but the statement for $\kappa \rightarrow-\infty$ follows similarly). We obtain

$$
F_{i, P}\left(2 \pi t \kappa e_{i}\right)=e^{-2 \pi \imath t \kappa\left\langle P, e_{i}\right\rangle} \kappa^{-\nu} c_{0}(P) e^{\frac{\pi i}{2 \nu} \operatorname{sign}(\kappa)}+O\left(\kappa^{-\nu-\eta}\right)
$$

where $c_{0}(P)>0$ and $\eta$ is the reciprocal of the least common multiple of $a_{1}, \ldots, a_{n}$. Thus

$$
I(t)=c_{0}(P) \pi^{-1} \sum_{\kappa>0}|\kappa|^{-\nu-1} \sin \left(2 \pi \kappa t\left\langle P, e_{i}\right\rangle-\pi /(2 \nu)\right)+O\left(\kappa^{-\nu-1-\eta}\right) .
$$

The sum defines a periodic function which is not identically zero, by the uniqueness theorem for Fourier series. Combining this with the estimation for the error term $I I(t)$ we see that $\lim \sup _{t \rightarrow \infty}\left|G_{P}(t)\right|>0$.

Remark. For almost all rotations the estimates for the error term improve. There is $r>2$ so that

$$
\left|E_{A \Omega}(t)\right| \leq \mathcal{C}(A) t^{d-1-\frac{d-1}{d+1}} \log ^{1 / r}(2+t)
$$

(indeed $\mathcal{C}$ is in $L^{q}(S O(d))$ for $\left.q<r\right)$. As in $[2]$ this is proved using a result on the maximal function

$$
M(\theta)=\sup _{r>0} r^{(d+1) / 2}|\widehat{\chi \Omega}(r \theta)|
$$

which was shown by Svensson [30] to be in $L^{q_{0}}\left(S^{d-1}\right)$ for some $q_{0}>2$ (under our assumption of finite line type, see also [25] for a similar result with additional real analyticity assumption). Indeed, let $\mathcal{R}_{\varepsilon, A}(t)=$ $\sum_{k \neq 0} \chi_{\Omega}(2 \pi t A k) \widehat{\zeta}(2 \pi \varepsilon t k)$ and

$$
\mathcal{M}_{j}(A)=\sup _{2^{j} \leq t \leq 2^{j+1}}\left|\mathcal{R}_{\varepsilon_{j}, A}(t)\right|, \quad \text { with } \varepsilon_{j}=2^{-2 j d /(d+1)}
$$

then for $q \leq q_{0}$

$$
\begin{aligned}
\left\|\mathcal{M}_{j}\right\|_{L^{q}(S O(d))} \leq & 2^{j d} \sum_{k \neq 0}\left(1+\left|\varepsilon_{j} 2^{j}\right| k \mid\right)^{-N}\left(2^{j}|k|\right)^{-(d+1) / 2} \\
& \times\left(\int\left|M\left(A \frac{k}{|k|}\right)\right|^{q} d A\right)^{1 / q} \\
& \lesssim 2^{j\left(d-1-\frac{d-1}{d+1}\right)}\|M\|_{L^{q}\left(S^{d-1}\right)}
\end{aligned}
$$


by the (standard) choice of $\varepsilon_{j}$. But

$$
\begin{aligned}
\left|E_{A \Omega}(t)\right| t^{-\left(d-1-\frac{d-1}{d+1}\right)} \log ^{-1 / r}(2+t) & \\
& \lesssim 1+\left(\sum_{j>0}\left|\mathcal{M}_{j}(A) 2^{-j\left(d-1-\frac{d-1}{d+1}\right)}(1+j)^{-1 / r}\right|^{q}\right)^{1 / q}
\end{aligned}
$$

which is in $L^{q}(S O(d))$ for $r<q_{0}$.

We remark that the methods in W. Müller's paper [22] could be used to improve the above bound to $\left|E_{A \Omega}(t)\right| \leq \mathcal{C}(A) t^{d-1-\frac{d-1}{d+1}-\beta}$ where $\beta=\beta(\Omega)>0$ and $\mathcal{C}$ is finite almost everywhere.

\section{Bochner-Riesz multipliers - the case of one nonvanishing principal curvature}

In this section we shall prove a general theorem concerning multipliers of Bochner-Riesz type associated to surfaces with at least one nonvanishing principal curvature. Then, in the subsequent section, we shall deduce Theorem 1.1 by rescaling arguments.

In what follows $M_{p}$ will be the space of Fourier multipliers on $L^{p}\left(\mathbb{R}^{d}\right)$; $\|m\|_{M_{p}}$ is the operator norm of the operator $T_{m}$ defined by $\widehat{T_{m} f}(\xi)=$ $m(\xi) \widehat{f}(\xi)$.

We split variables in $\mathbb{R}^{d}$ as $\xi=\left(\widetilde{\xi}, \xi_{d}\right)$ and in the statement of the proposition we further split $\widetilde{\xi}=\left(\xi_{1}, \xi^{\prime}\right) \in \mathbb{R} \times \mathbb{R}^{d-2}$. The proof of the following result uses the ideas from the two-dimensional case, see $[\mathbf{9}],[\mathbf{6}]$.

Proposition 4.1. Let $\varepsilon>0, N \geq d+1+2 / \varepsilon$, and let $g \in C^{N}\left(\mathbb{R}^{d-1}\right)$. Suppose that there is a cube $U$ centered at the origin and $a>0$ so that

$$
\frac{\partial^{2} g}{\partial \xi_{1}^{2}}\left(\xi_{1}, \xi^{\prime}\right) \geq a
$$

in $U$. Let $\chi$ be supported in $U$ and let $\phi$ be a smooth function supported in $(1 / 2,2)$. Let $0<\delta \ll 1$ and

$$
m_{\delta}(\xi)=\chi(\xi) \phi\left(\delta^{-1}\left(\xi_{d}-g\left(\xi_{1}, \xi^{\prime}\right)\right)\right) .
$$

Then

$$
\left\|m_{\delta}\right\|_{M_{4}} \leq C_{\varepsilon} \delta^{-\frac{d-2}{4}-\varepsilon},
$$

where $C_{\varepsilon}$ depends only on $a, \varepsilon, U$, the $C^{N}(U)$ norms of the functions $g$, $\chi$ and the $C^{d+1}$ norm of $\phi$. 
Proof: We may assume that $U$ is the unit cube, and that the support of $\chi$ has small diameter. We decompose $m_{\delta}=\sum_{k} m_{\delta, k}$ where $k=$ $\left(k_{2}, \ldots, k_{d-1}\right)$ ranges over $(d-2)$-tuples of integers $k_{i} \leq C \delta^{-1 / 2}$ and

$$
m_{\delta, k}(\xi)=m_{\delta}(\xi) \prod_{i=2}^{d-1} \psi\left(\delta^{-1 / 2} \xi_{i}-k_{i}\right)
$$

for suitable $\psi \in C_{0}^{\infty}$ satisfying $\sum_{n=-\infty}^{\infty} \psi(s-n)=1$, so that $\operatorname{supp} \psi \subset$ $[-1,1]$. Let $\widetilde{\psi} \in C_{0}^{\infty}([-2,2])$ so that $\widetilde{\psi}$ is equal to 1 on the support of $\psi$.

Denote by $T_{k}$ the convolution operator with Fourier multiplier $m_{\delta, k}$ and by $R_{k}$ the convolution operator with Fourier multiplier $\widetilde{\psi}\left(\delta^{-1 / 2} \xi^{\prime}-\right.$ $k)$. Note that $\left\|R_{k}\right\|_{L^{p} \rightarrow L^{p}} \leq C, 1 \leq p \leq \infty$. Then for $2 \leq p \leq \infty$

$$
\left\|\sum_{k} R_{k} g_{k}\right\|_{p} \lesssim\left(\sum_{k}\left\|g_{k}\right\|_{p}^{p^{\prime}}\right)^{1 / p^{\prime}}
$$

which follows for $p=\infty$ from Minkowski's inequality and for $p=2$ by orthogonality; for $2<p<\infty$ one uses interpolation. Since $T_{k}=R_{k} T_{k} R_{k}$ it follows that

$$
\left\|\sum_{k} T_{k}\right\|_{L^{4} \rightarrow L^{4}} \leq C \delta^{-(d-2) / 4} \sup _{k}\left\|T_{k}\right\|_{L^{4} \rightarrow L^{4}}
$$

and therefore it suffices to show that

$$
\left\|T_{k}\right\|_{L^{4} \rightarrow L^{4}} \lesssim \delta^{-\varepsilon}
$$

The estimate (4.1) is proved using arguments in [6] which we will sketch. For $\nu \in \mathbb{Z}$ we define operators $T_{k, \nu}$ and $S_{\nu}$ by $\widehat{S_{\nu} f}(\xi)=\widetilde{\psi}\left(\delta^{-1 / 2} \xi_{1}-\right.$ $\nu)$ and $\widehat{T_{k, \nu} f}(\xi)=\psi\left(\delta^{-1 / 2} \xi_{1}-\nu\right) \widehat{T_{k} f}(\xi)$. Then $T_{k}=\sum_{\nu} T_{k, \nu} S_{\nu} f$ where the sum is extended over integers $\nu$ with $|\nu| \ll \delta^{-1 / 2}$ since we assume that the support of $\chi$ is small.

Now

$$
\left\|\sum_{\nu} T_{k, \nu} S_{\nu} f\right\|_{4}^{2}=\left\|\sum_{\nu, \nu^{\prime}}\left(T_{k, \nu} S_{\nu} f\right)\left(T_{k, \nu^{\prime}} S_{\nu^{\prime}} f\right)\right\|_{2}
$$

$$
\leq \sum_{\ell: 2^{\ell} \delta^{1 / 2} \ll 1}\left\|\sum_{\substack{\left(\nu, \nu^{\prime}\right): \\ \nu-\nu^{\prime} \mid \approx 2^{\ell}}}\left(T_{k, \nu} S_{\nu} f\right)\left(T_{k, \nu^{\prime}} S_{\nu^{\prime}} f\right)\right\|_{2} .
$$


It can be checked that the family of functions $\left(T_{k, \nu} S_{\nu} f\right)\left(T_{k, \nu^{\prime}} S_{\nu^{\prime}} f\right)$ has an orthogonality property which implies that

$$
\left\|\sum_{\substack{\left(\nu, \nu^{\prime}\right) \\\left|\nu-\nu^{\prime}\right| \approx 2^{\ell}}}\left(T_{k, \nu} S_{\nu} f\right)\left(T_{k, \nu^{\prime}} S_{\nu^{\prime}} f\right)\right\|_{2} \lesssim\left\|\left(\sum_{\nu}\left|T_{k, \nu} S_{\nu} f\right|^{2}\right)^{1 / 2}\right\|_{4}^{2}
$$

The proof of (4.3) is based on an idea of C. Fefferman [9]; in higher dimensions one uses the following

Lemma 4.2. Suppose that $a^{\prime} \in \mathbb{R}^{d-2},\left|a^{\prime}\right| \ll 1$, and the vectors $\widetilde{\xi}, \widetilde{\eta}, \widetilde{\zeta}$, $\widetilde{\omega}$ satisfy

(i) $\xi+\eta-\widetilde{\zeta}-\widetilde{\omega}=0$,

(ii) $\xi_{1}>\zeta_{1}>0, \eta_{1}<\omega_{1}<0$,

(iii) $|\widetilde{\xi}|,|\widetilde{\eta}|,|\widetilde{\zeta}|,|\widetilde{\omega}| \in\left[2^{\ell-1} \delta^{1 / 2}, 2^{\ell+1} \delta^{1 / 2}\right]$,

(iv) $\xi^{\prime}, \eta^{\prime}, \zeta^{\prime}$ and $\omega^{\prime}$ belong to the cube of sidelength $4 \delta^{1 / 2}$ centered at $a^{\prime}$.

Then

$$
g(\widetilde{\xi})+g(\widetilde{\eta})-g(\widetilde{\zeta})-g(\widetilde{\omega}) \geq c 2^{\ell} \delta^{1 / 2}\left(\left|\xi_{1}-\zeta_{1}\right|+\left|\eta_{1}-\omega_{1}\right|\right) .
$$

In (4.4), $c$ depends only on the lower bound of $g_{\xi_{1}} \xi_{1}$ and the $C^{4}$ norm of $g$ in $\operatorname{supp} \chi$.

Sketch of Proof: A Taylor expansion about the origin yields

$$
g(\widetilde{\xi})+g(\widetilde{\eta})-g(\widetilde{\zeta})-g(\widetilde{\omega})=I+I I+I I I+I V
$$

where

$$
\begin{aligned}
I & =\frac{1}{2} g_{\xi_{1} \xi_{1}}(0)\left(\xi_{1}^{2}+\eta_{1}^{2}-\zeta_{1}^{2}-\omega_{1}^{2}\right) \\
I I & =\frac{1}{2}\left(\xi_{1}\left\langle g_{\xi_{1} \xi^{\prime}}(0), \xi^{\prime}\right\rangle+\eta_{1}\left\langle g_{\xi_{1} \xi^{\prime}}(0), \eta^{\prime}\right\rangle-\zeta_{1}\left\langle g_{\xi_{1} \xi^{\prime}}(0), \zeta^{\prime}\right\rangle-\omega_{1}\left\langle g_{\xi_{1} \xi^{\prime}}(0), \omega^{\prime}\right\rangle\right) \\
I I I & =\frac{1}{2}\left(\left\langle\xi^{\prime}, g_{\xi^{\prime} \xi^{\prime}}(0) \xi^{\prime}\right\rangle+\left\langle\eta^{\prime}, g_{\xi^{\prime} \xi^{\prime}}(0) \eta^{\prime}\right\rangle-\left\langle\zeta^{\prime}, g_{\xi^{\prime} \xi^{\prime}}(0) \zeta^{\prime}\right\rangle-\left\langle\omega^{\prime}, g_{\xi^{\prime} \xi^{\prime}}(0) \omega^{\prime}\right\rangle\right) \\
I V & =r(\widetilde{\xi})+r(\widetilde{\eta})-r(\widetilde{\zeta})-r(\widetilde{\omega})
\end{aligned}
$$


where $r$ vanishes of third order at the origin. (4.4) is proved by verifying

$$
\begin{aligned}
I & \approx 2^{\ell} \delta^{1 / 2}\left(\left|\xi_{1}-\zeta_{1}\right|+\left|\eta_{1}-\omega_{1}\right|\right) \\
I I & \leq C 2^{\ell} \delta \\
I I I & \leq C \delta \\
I V & \leq C 2^{2 \ell} \delta\left(\left|\xi_{1}-\zeta_{1}\right|+\left|\eta_{1}-\omega_{1}\right|\right) .
\end{aligned}
$$

The straightforward calculation is omitted; we note that formula (6.30) in $[\mathbf{2 1}]$ turns out to be useful in order to carry it out.

Proof of Proposition 2.1, cont.: By (4.3) it remains to show that

$$
\left\|\left(\sum_{\nu}\left|T_{k, \nu} S_{\nu} f\right|^{2}\right)^{1 / 2}\right\|_{4} \lesssim \delta^{-\varepsilon}\|f\|_{4} .
$$

Let $\Gamma_{k}(t)=\left(-\nabla_{\widetilde{\xi}} g\left(t, \delta^{1 / 2} k\right), 1\right)$ which gives a one parameter family of vectors normal to $\Sigma_{\rho}$.

For $\sigma \geq 2$ let $\mathcal{R}_{k, \sigma}$ be the set of all cylinders whose base is a $d-2$ dimensional ball of radius $s$ and whose height is $\sigma s$ (any $s>0$ ), so that the axis is parallel to $\Gamma_{k}(t)$ for some $|t| \leq 1$.

Define the maximal function

$$
M_{k, \sigma} f(x)=\sup _{\substack{x \in R \\ R \in \mathcal{R}_{k, \sigma}}} \frac{1}{|R|} \int_{R}|f(y)| d y .
$$

Then arguing as in $[\mathbf{6}]$ and using standard estimates for the kernel of $T_{k, \nu}$ we see that

$$
\int \sum_{\nu}\left|T_{k, \nu} S_{\nu} f(x)\right|^{2} w(x) d x \lesssim \int \sum_{\nu}\left|S_{\nu} f(x)\right|^{2} M_{k, \delta^{-1 / 2}} w(x) d x .
$$

The $L^{p}$ norm of $\left(\sum_{\nu}\left|S_{\nu} f\right|^{2}\right)^{1 / 2}$ is bounded by the $L^{p}$ norm of $f$, for $p \geq 2$ (see [6]) and therefore we can finish our proof by using duality and showing that

$$
\left\|M_{k, \sigma} f\right\|_{2} \leq C_{\varepsilon} \sigma^{\varepsilon}\|f\|_{2}
$$

uniformly in $k$.

If we knew that for every $\xi$ the function $t \mapsto\left\langle\xi, \Gamma_{k}(t)\right\rangle$ changed sign at most $M$ times then it would follow from a result by Córdoba $[\mathbf{7}]$ that (4.6) holds with $\sigma^{\varepsilon}$ replaced by $C_{1} M[\log \sigma]^{C_{2}}$. This hypothesis may not be satisfied, but we can get around this point by a simple approximation. Namely, divide $[-1,1]$ into $\sigma^{\varepsilon / 2}$ intervals $\left[a_{j}, b_{j}\right]$ of lengths $\sigma^{-\varepsilon / 2}$. 
Let $P_{k, j}(t)$ be the vector valued Taylor polynomial of degree $[2 / \varepsilon]$ of $\nabla_{\widetilde{\xi}} g\left(\cdot, \delta^{1 / 2} k\right)$ expanded about $a_{j}$, and let $\Gamma_{k, j}(t)=\left(-P_{k, j}(1), 1\right)$. Then $\left|\Gamma_{k}(t)-\Gamma_{k, j}(t)\right| \leq C \sigma^{-1}$ for $t \in\left[a_{j}, b_{j}\right]$.

Let $\mathcal{R}_{k, \sigma, j}$ be the set of all cylinders whose base is a $d$-2-dimensional ball of radius $s$ whose height is $\sigma s$, so that the axis is parallel to $\Gamma_{k, j}(t)$ for some $|t| \leq 1$. If $M_{k, \sigma, j}$ denotes the associated maximal operator then it is immediate that $M_{k, \sigma} f \leq \sum_{j} M_{k, \sigma, j} f$ where the sum contains only $O\left(\sigma^{\varepsilon / 2}\right)$ terms. Córdoba's result yields the $L^{2}$ bound $C_{\varepsilon}[\log \sigma]^{C_{2}}$ for each $M_{k, \sigma, j}$. This finishes the proof of (4.6).

\section{Proof of Theorem 1.1}

The $L^{1}$ version of the theorem is well known, and therefore by an interpolation argument one has to show the boundedness on $L^{4 / 3}\left(\mathbb{R}^{d}\right)$, or, equivalently, on $L^{4}\left(\mathbb{R}^{d}\right)$.

We split $(1-\rho(\xi))_{+}^{\lambda}=h_{0}(\rho(\xi))+h_{1}(\rho(\xi))$ where $h_{0}$ is supported in $\left\{t: t \leq 1-\epsilon_{0}\right\}$ for suitable small $\epsilon_{0}$ and $h_{1}$ is supported in $\left\{t: t>1-2 \epsilon_{0}\right\}$. Then $h_{0}(\rho(\xi))$ is a Fourier multiplier in $M_{1}$; the mild singularity at the origin can be handled e.g. by an averaging argument in [8, p. 248], replacing $\rho$ by $\rho^{N}$ for large $N$.

Let $\xi^{0} \in \Sigma_{\rho}$. It suffices to show that there exists a neighborhood $V$ of $\xi^{0}$ (in $\mathbb{R}^{d}$ ) so that $h_{1}(\rho(\xi)) \tilde{\chi}$ is a multiplier on $\mathbb{R}^{d}$ for $\lambda>(d-2) / 4$ if $\tilde{\chi} \in C^{\infty}$ and supported in $V$. The multiplier norm is invariant under rotations and we may assume that $\Sigma_{\rho}$ can be parametrized as a graph $\xi_{d}=G(\widetilde{\xi}), \widetilde{\xi} \in \mathbb{R}^{d-1}$ near $\xi^{0}$, so that $\rho(\xi)<1$ if $\xi_{d}>G(\widetilde{\xi})$. We write

$$
\chi(\xi) h_{1}(\rho(\xi))=\chi(\xi) H(\xi)\left(\xi_{d}-G(\widetilde{\xi})\right)_{+}^{\lambda} \quad \text { where } H(\xi)=\left(\frac{1-\rho(\xi)}{\xi_{d}-G(\widetilde{\xi})}\right)^{\lambda} .
$$

A Taylor expansion of $\rho$ about $\xi_{d}=G(\widetilde{\xi})$ shows that $H$ is smooth on supp $\chi$; therefore by the algebra property of $M_{p}$ it suffices to show that $\tilde{\chi}(\xi)\left(\xi_{d}-G\left(\xi_{1}, \xi^{\prime}\right)\right)_{+}^{\lambda}$ belongs to $M_{4}$ if $\operatorname{supp} \tilde{\chi}$ is sufficiently close to $\xi^{0}$.

Let $\mathfrak{a}=\left(a_{1}, \ldots, a_{d-1}\right)$ be the multitype of $\Sigma_{\rho}$ at $\xi^{0}$, in the sense of Subsection 1.2. By an affine transformation we may assume that $\xi^{0}=0$, $G(0)=\nabla G(0)=0$, and that $G=Q+R$ where $Q$ and $R$ are as in the proof of Lemma 2.2: The function $Q$ is mixed homogeneous of degree $\left(a_{1}, \ldots, a_{d-1}\right)$, i.e. if $A_{s}(\widetilde{\xi})=\left(s^{\frac{1}{a_{1}}} \xi_{1}, \ldots, s^{\frac{1}{a_{d-1}}} \xi_{d-1}\right)$ then $Q$ satisfies $Q\left(A_{s}(\widetilde{\xi})\right)=s Q(\widetilde{\xi})$. The remainder term $R$ satisfies $\left|s^{-1} \frac{\partial^{|\alpha|}}{\partial \xi^{\alpha}}\left(R\left(A_{s} \widetilde{\xi}\right)\right)\right| \leq$ $C_{M, N} s^{1 / m}$ for small $x$ and $s$ and all multiindices $\alpha=\left(\alpha_{1}, \ldots, \alpha_{d-1}\right)$ with 
$|\alpha| \leq N$. In particular $|R(\widetilde{\xi})| \leq Q(\widetilde{\xi}) / 10$ if $Q(\widetilde{\xi}) \leq 2^{-r_{0}+2}$ for suitably large $r_{0}$.

Next we set $R_{r}(\widetilde{\xi})=2^{r} R\left(A_{2^{-r}} \widetilde{\xi}\right)$, so that $G_{r}=Q+R_{r}$ tends to $G$ in the $C^{\infty}$ topology, as $r \rightarrow \infty$. Since the Hessian of $Q$ has rank 1 where $1 / 4<Q(\widetilde{\xi}) \leq 4$ (see $(2.10)$ ), the same is true for $G_{r}=Q+R_{r}$ if $r$ is large; we may assume that the matrix norm of $\left(Q+R_{r}\right)^{\prime \prime}$ is bounded below uniformly in $r$ if $r \geq r_{0}$.

Let $\phi_{1}$ be supported in $(1 / 2,2)$ such that $\sum_{k \geq 0} \phi_{1}\left(2^{k} s\right)=1$ for $0<$ $s \leq 1$. Then we have to show a bound for the $M_{4}^{-}$norm of

$$
\kappa_{j}(\xi)=\widetilde{\chi}(\xi) \phi_{1}\left(2^{j}\left(\xi_{d}-G\left(\xi_{1}, \xi^{\prime}\right)\right)\right)\left(\xi_{d}-G\left(\xi_{1}, \xi^{\prime}\right)\right)_{+}^{\lambda} .
$$

Here we may assume that $\widetilde{\chi}(\xi)=0$ when $Q(\widetilde{\xi}) \geq 2^{-r_{0}}$.

We now perform a further decomposition in terms of $G(\widetilde{\xi})$. Let $\eta \in$ $C_{0}^{\infty}(\mathbb{R})$ so that $\eta(s)=1$ if $|s| \leq 1 / 2$ and $\eta(s)=0$ if $|s| \geq 1$; also let $\eta_{0}=\eta$ and for integer $r>0$ let $\eta_{r}(s)=\eta\left(2^{-r} s\right)-\eta\left(2^{-r+1} s\right)$. Let

$$
\kappa_{j, n}(\xi)=\kappa_{j}(\xi) \eta_{n}\left(2^{j} G(\widetilde{\xi})\right)
$$

so that $\kappa_{j, n}$ is supported where $\left|\xi_{d}-G(\widetilde{\xi})\right| \approx 2^{-j}$ and $G(\widetilde{\xi}) \approx 2^{n-j}$ if $n \geq 0$ and $G(\widetilde{\xi}) \lesssim 2^{-j}$ if $n=0$. Using the assumption on the support of the cutoff function $\tilde{\chi}$ we see that $\kappa_{j, n}=0$ for $j \leq n+r_{0}$.

For the pieces $\kappa_{j, n}$ we employ a scaling argument (for a similar argument in two dimensions see [12]). For the scaling we use the dilations $\xi \mapsto\left(A_{2^{n-j}}(\widetilde{\xi}), 2^{n-j} \xi_{d}\right)$. Define for $n>0$

$\widetilde{\kappa}_{j, n}\left(\widetilde{\xi}, \xi_{d}\right)=\phi_{1}\left(2^{n}\left(\xi_{d}-G_{j-n}\left(\xi_{1}, \xi^{\prime}\right)\right)\right)\left(\xi_{d}-G_{j-n}\left(\xi_{1}, \xi^{\prime}\right)\right)_{+}^{\lambda} \eta_{1}\left(G_{j-n}(\widetilde{\xi})\right) ;$

for $n=0$ we use the same formula but with $\eta_{1}$ replaced by $\eta=\eta_{0}$. Then

$$
\kappa_{j, n}\left(A_{2^{n-j}} \widetilde{\xi}, 2^{n-j} \xi_{d}\right)=2^{(n-j) \lambda} \widetilde{\chi}\left(A_{2^{n-j}} \widetilde{\xi}, 2^{n-j} \xi_{d}\right) \widetilde{\kappa}_{j, n}\left(\widetilde{\xi}, \xi_{d}\right)
$$

so that

$$
\left\|\kappa_{j, n}\right\|_{M_{p}} \lesssim 2^{(n-j) \lambda}\left\|\widetilde{\kappa}_{j, n}\right\|_{M_{p}} .
$$

It is now easy to see that the $C^{4}$ norm of $\widetilde{\kappa}_{j, 0}$ is $\lesssim 2^{-j \lambda}$ and $\widetilde{\kappa}_{j, 0}$ is supported in a fixed ball with diameter independent of $j$.

Therefore

$$
\left\|\widetilde{\kappa}_{j, 0}\right\|_{M_{p}} \lesssim 2^{-j \lambda}, \quad 1 \leq p \leq \infty
$$


Note that for $j-n \geq r_{0}$ the multipliers $\widetilde{\kappa}_{j, n}$ are supported where $1 / 4<Q(\widetilde{\xi})<4$, and by construction the matrix norm of $G_{j-n}^{\prime \prime}$ is in this region bounded above and below, for $j-n \geq r_{0}$. We may apply Proposition 4.1 (with $\delta=2^{-n}$ ), to see that for $0<n \leq j-r_{0}$

$$
\left\|\widetilde{\kappa}_{j, n}\right\|_{M_{4}} \lesssim 2^{(n-j) \lambda} 2^{-n\left(\lambda-\frac{d-2}{4}\right)}
$$

and the assertion of Theorem 1.1 follows by summing over $0<n \leq j-r_{0}$, $j>0$.

\section{References}

[1] J. Bourgain, Besicovitch type maximal operators and applications to Fourier analysis, Geom. Funct. Anal. 1(2) (1991), 147-187.

[2] L. Brandolini, L. Colzani, A. Iosevich, A. Podkorytov and G. Travaglini, Geometry of the Gauss map and lattice points in convex domains, Mathematika (to appear).

[3] J. Bruna, A. Nagel and S. Wainger, Convex hypersurfaces and Fourier transforms, Ann. of Math. (2) 127(2) (1988), 333-365.

[4] L. Carleson And P. SJÖlin, Oscillatory integrals and a multiplier problem for the disc, Studia Math. 44 (1972), 287-299.

[5] Y. Colin de Verdière, Nombre de points entiers dans une famille homothétique de domains de R, Ann. Sci. École Norm. Sup. (4) 10(4) (1977), 559-575.

[6] A. CórdobA, A note on Bochner-Riesz operators, Duke Math. J. 46(3) (1979), 505-511.

[7] A. CóRdoba, Geometric Fourier analysis, Ann. Inst. Fourier (Grenoble) 32(3) (1982), 215-226.

[8] H. Dappa and W. Trebels, On maximal functions generated by Fourier multipliers, Ark. Mat. 23(2) (1985), 241-259.

[9] C. Fefferman, A note on spherical summation multipliers, Israel J. Math. 15 (1973), 44-52.

[10] A. Greenleaf, Principal curvature and harmonic analysis, Indiana Univ. Math. J. 30(4) (1981), 519-537.

[11] E. HlawKA, Über Integrale auf konvexen Körpern. I, Monatsh. Math. 54 (1950), 1-36; Integrale auf konvexen Körpern. II, Monatsh. Math. 54 (1950), 81-99.

[12] L. Hörmander, Oscillatory integrals and multipliers on $F L^{p}$, Ark. Mat. 11 (1973), 1-11.

[13] L. Hörmander, 'The analysis of linear partial differential operators. I. Distribution theory and Fourier analysis", Grundlehren 
der Mathematischen Wissenschaften 256, Springer-Verlag, Berlin, 1983.

[14] M. N. HuXLEY, "Area, lattice points, and exponential sums", London Mathematical Society Monographs. New Series 13, Oxford Science Publications, The Clarendon Press, Oxford University Press, New York, 1996.

[15] A. Iosevich, Lattice points and generalized Diophantine conditions, J. Number Theory 90(1) (2001), 19-30.

[16] A. Iosevich And E. SaWyer, Maximal averages over surfaces, Adv. Math. 132(1) (1997), 46-119.

[17] A. Iosevich, E. Sawyer and A. Seeger, On averaging operators associated with convex hypersurfaces of finite type, J. Anal. Math. 79 (1999), 159-187.

[18] E. KRäTzEL, "Lattice points", Mathematics and its Applications (East European Series) 33, Kluwer Academic Publishers Group, Dordrecht, 1988.

[19] E. Krätzel and S. Hoeppner, The number of lattice points inside and on the surface $\left|t_{1}\right|^{k}+\left|t_{2}\right|^{k}+\cdots+\left|t_{n}\right|^{k}=x$, Math. Nachr. 163 (1993), 257-268.

[20] E. KRÄtzel And W. G. NowaK, Georg lattice points in large convex bodies, Monatsh. Math. 112(1) (1991), 61-72; Lattice points in large convex bodies. II, Acta Arith. 62(3) (1992), 285-295.

[21] G. Mockenhaupt, A. Seeger and C. D. Sogge, Local smoothing of Fourier integral operators and Carleson-Sjölin estimates, $J$. Amer. Math. Soc. 6(1) (1993), 65-130.

[22] W. MüLLER, Lattice points in large convex bodies, Monatsh. Math. 128(4) (1999), 315-330.

[23] W. G. NowAK, Zur Gitterpunktlehre der euklidischen Ebene, Akad. Wetensch. Indag. Math. 46(2) (1984), 209-223; Zur Gitterpunktlehre der euklidischen Ebene. II, Österreich. Akad. Wiss. Math.-Natur. Kl. Sitzungsber. II 194(1-3) (1985), 31-37.

[24] B. Randol, A lattice-point problem, Trans. Amer. Math. Soc. 121 (1966), 257-268.

[25] B. RANDOL, On the Fourier transform of the indicator function of a planar set, Trans. Amer. Math. Soc. 139 (1969), 271-278; On the asymptotic behavior of the Fourier transform of the indicator function of a convex set, Trans. Amer. Math. Soc. 139 (1969), 279-285.

[26] H. Schulz, Convex hypersurfaces of finite type and the asymptotics of their Fourier transforms, Indiana Univ. Math. J. 40(4) (1991), $1267-1275$. 
[27] A. Seeger and S. Ziesler, Riesz means associated with convex domains in the plane, Math. Z. 236(4) (2001), 643-676.

[28] P. SJöLIN, Fourier multipliers and estimates of the Fourier transform of measures carried by smooth curves in $R^{2}$, Studia Math. $\mathbf{5 1}$ (1974), 169-182.

[29] E. M. Stein, "Harmonic analysis: real-variable methods, orthogonality, and oscillatory integrals", Princeton Mathematical Series 43, Monographs in Harmonic Analysis III, Princeton University Press, Princeton, NJ, 1993.

[30] I. Svensson, Estimates for the Fourier transform of the characteristic function of a convex set, Ark. Mat. 9 (1971), 11-22.

[31] M. Tarnopolska-Weiss, On the number of lattice points in planar domains, Proc. Amer. Math. Soc. 69(2) (1978), 308-311.

[32] A. N. Varchenko, The number of lattice points in families of homothetic domains in $\mathbf{R}^{n}$, Funktsional. Anal. i Prilozhen. 17(2) (1983), 1-6.

\section{Alexander Iosevich:}

Mathematics Department

University of Missouri

Columbia, MO 65211

U.S.A.

E-mail address: iosevich@wolff.math.missouri.edu

Eric Sawyer:

Department of Mathematics and Statistics

1280 Main Street West

Hamilton, Ontario L8S 4K1

Canada

E-mail address: sawyer@mcmaster.ca

Andreas Seeger:

Department of Mathematics

University of Wisconsin-Madison

Madison, WI 53706

U.S.A.

E-mail address: seeger@math.wisc.edu 